than 2 years old, were interviewed in this controlled before and after, cross-sectional study.

Results After the intervention the proportion of women, during their last pregnancy, attending antenatal care, at least once, increased by from $84.6 \%$ to $96.8 \%(p=0.00)$. The proportion of women who had their first prenatal visit in the first trimester was increased from $39.9 \%$ to $58.1 \%(p=0.00)$. Women attending more than 4 visits increased from $37.1 \%$ to $42.6 \%(p=0.28)$.

Conclusion This ongoing community intervention providing health promotion shows an improvement on women's attendance of antenatal care.

\section{PS22 LIFE COURSE SOCIO-ECONOMIC POSITION AND QUALITY OF LIFE IN ADULTHOOD: A SYSTEMATIC REVIEW OF LIFE COURSE MODELS}

doi:10.1136/jech-2012-201753.121

${ }^{1} \mathrm{C}$ Niedzwiedz, ${ }^{2}$ SV Katikireddi, 'J Pell, 'R Mitchell. 'Institute of Health \& Wellbeing, University of Glasgow, Glasgow, UK; ${ }^{2}$ Evaluating the Health Effects of Social Interventions Programme, MRC/CSO Social \& Public Health Sciences Unit, Glasgow, UK

Background Measurement and determinants of quality of life are of increasing interest to researchers and policymakers. A relationship between current socio-economic position and subjective quality of life has been demonstrated, using wellbeing, life and needs satisfaction approaches. Less is known regarding the influence of different socio-economic trajectories across the life course and their subsequent effect on quality of life. Several conceptual models have been proposed to help explain potential life course effects on health, including accumulation, latent, pathway and social mobility models. This systematic review aimed to apply these models to studies investigating life course socio-economic effects on quality of life, in order to assess which model(s) best described any relationship.

Methods A review protocol was developed detailing explicit inclusion and exclusion criteria, search terms, data extraction items and quality appraisal procedures. Literature searches were performed in 12 electronic databases during January 2012 and the references and citations of included articles were checked for additional relevant articles. Narrative synthesis was used to analyse the results and studies were categorised into groups based on the life course design implemented.

Results After screening 7,566 records, 12 studies met the eligibility criteria. The included articles used data from 10 different datasets and five countries. Study quality varied and heterogeneity was high. Five studies assessed the latent model, two assessed the pathway model and three tested the accumulation model. Seven studies assessed social mobility models (inter-generational mobility in three, intra-generational mobility in five and one included both types). More evidence was found to support the latent model among women (but results were contradictory) and mixed evidence was found for intra-generational mobility and pathway effects. Associations also tended to vary by gender. Few studies were identified for accumulation and inter-generational mobility effects and heterogeneity of these studies resulted in limited synthesis.

Conclusion A lack of available and comparable evidence prevented the identification of the optimal life course model(s). Different target populations, outcomes and methodologies used between studies likely contributed to the mix of results. To improve the potential for synthesis in this area, future studies should aim to increase study comparability. Recommendations include testing all life course models within studies and the use of multiple measures of socioeconomic position and quality of life. To enable investigation of between-country differences, the increased collection, harmonisation and utilisation of comparable cross-national data would be beneficial.

\section{PS23 HOW WELL DOES THE NHS SUPPORT EMPLOYEES WHO WISH TO BREASTFEED ON RETURN TO WORK?}

doi:10.1136/jech-2012-201753.122

${ }^{1} \mathrm{LI}$ Watson, ${ }^{2} \mathrm{~J}$ Neufeind, ${ }^{2} \mathrm{E}$ George, ${ }^{3}$ Fenske. ${ }^{1}$ Department of Public Health, NHS Fife, Leven, UK; ${ }^{2}$ Playfield Institute, NHS Fife, Cupar, UK; ${ }^{3}$ Victoria Hospital, NHS Fife, Kirkcaldy, UK

Background In line with EU and UK law, NHS organisations have policies to support employees who wish to continue breastfeeding on return to work, which has numerous health benefits. The NHS employs many women of reproductive age, but anecdotally some face significant barriers in continuing breastfeeding. The objectives of the study were to describe awareness of relevant policies, describe breastfeeding behaviours and experience of staff in relation to these, and identify any areas for improvement. Fife NHS Board provides full hospital and primary care services in southeast Scotland and employs 8,000 staff.

Methods A questionnaire survey with quantitative and qualitative elements was sent from the Department of Human Resources to eligible employees of NHS Fife who had been on maternity leave between 2007-09. This was adapted from previous surveys with input from experts and was piloted in two stages. Those with serious complications e.g. stillbirth were excluded. Full ethical review was not required.

Results 651 women were eligible, with 87 questionnaires undelivered, and 342/564 (61\%) returned. Respondents were comparable to female employees of NHS Fife.; 203/329 (62\%) reported awareness of the breastfeeding and return to work policy. 26/342 (8\%) reported being offered information on support breastfeeding on return to work. $63 / 270(23 \%)$ who breastfed at all continued on their return and this was more common with increasing age, but not related to setting eg ward, community. 18/270 (7\%) would have breastfed longer if their employer had been more supportive, and 43/270 (16\%) cited returning to work as a reason they stopped.; 102/254 (40.2\%) felt not very or not at all confident to approach their employer regarding support. Women who were aware of the policies were significantly more likely to feel confident than women who were not, $(p<0.001) .4$ respondents used a suitable area to express milk and 3 made use of a designated fridge for storage.

Key themes described were that it was perceived that managers and other workers grudged the extra time for expressing milk or breastfeeding, and lack of facilities.

Conclusion Experience of staff was highly variable suggesting the policy was not implemented consistently. Suggested areas for improvement include: clearer information regarding policies with specific information on support for continued breastfeeding, line managers initiating discussion regarding the possibility of breastfeeding on return to work, and ensuring that women who do continue are not stigmatised by colleagues.

While these results may not be fully generalisable, it is likely that there are similar issues for NHS employees more widely.

\section{PS24 NUTRITIONAL STATUS IN RELATION TO CARIES EXPERIENCE AMONG PRIMARY SCHOOL IRAOI CHILDREN IN THE MIDDLE REGION OF IRAQ}

doi:10.1136/jech-2012-201753.123

'Ban S Diab, 'SK El Samarrai, 'WS Al-Alousi, ${ }^{2} \mathrm{ASD}$ Al-Radha. ${ }^{1}$ College of Dentistry, University of Baghdad, Baghdad, Iraq; ${ }^{2}$ College of Dentistry, Al- Mustansiriyah University, Baghdad, Iraq

Background Malnutrition when occurs during development period may cause irreversible effect on developing dental tissues leading to dental caries. The aims of the present study were to evaluate the nutritional status of primary school children and its influence on dental caries. 
Methods This study was conducted among primary school children (6-10 years old) in the middle region of Iraq (five governorates). The total sample composed of 4,089 children randomly selected. The assessment of nutritional status was performed using height for age indicator for chronic malnutrition (stunted), the values were compared with the international reference values defined by the National Center for Health Statistics (NCHS) and classify them into mild, moderate, and sever stunted. The nutrient analysis was done through the dietary assessment using semi-quantitative food frequency questionnaires the assessment was converted to nutrient data (according to nutrient composition of Iraqi food) which was performed using software program especially designed for this study. Diagnosis and recording of dental caries was conducted according to World Health Organization criteria in 1997 using dmfs and DMFs indices for primary and permanent teeth respectively. Statistical analyses were performed using Analysis of Variance and Least Significant Different tests.

Results The prevalence of stunted, was found to be $49 \%$, where the mild grade was the most prevalent (29\%). The data presented in the study showed that there is a significant difference in mean of caries experience between different grades of nutritional status $(\mathrm{P}<0.01)$. The well-nourished children had significantly lower $\mathrm{dmfs}$ values $(11.76 \pm 0.95)$ than mild $(10.44 \pm 0.61)$ and sever stunted (16.32 \pm 1.07$)$. Concerning caries experience of permanent dentition, apposite figure were found, well-nourished children had higher DMFS values $(2.11 \pm 0.93)$ than mild $(1.39 \pm 0.07)$, moderate $(1.56 \pm 0.10)$, and sever stunted (1.14 \pm 0.18$)$. However all these differences were statistically not significant $(\mathrm{P}>0.05)$

The daily nutrients intake for the well-nourished children were recorded to be for protein $52.0 \pm 0.62$ grams, calcium $0.63 \pm 0.01$ grams, phosphorus $1.01 \pm 0.01$ grams, calcium/phosphate ratio $0.61 \pm 0.004$, iron $0.01 \pm 0.0002$ milligrams, vitamin A $6981 \pm 84.04$ I.U and vitamin C $0.10 \pm 0.002$ milligrams. These amounts tended to be decreased significantly with increased severity of malnutrition. Multiple linear regressions showed correlation between nutrients and dmfs, DMFs with $\mathrm{R}^{2}$ value $0.7 \%, 0.5 \%$ respectively.

Conclusion The oral cavity is considered a mirror of nutritional status of the body. Stunted children with inadequate nutrients intake during growth and development had a significant effect on tooth development and subsequent caries initiation and progression.

\section{PS25 DISCONTINUATION OF ANTIEPILEPTIC DRUGS IN PREGNANCY: A UK POPULATION BASED STUDY IN THE HEALTH IMPROVEMENT NETWORK (THIN)}

doi:10.1136/jech-2012-201753.124

'S Man, II Petersen, '2M Thompson, II Nazareth. 'Primary Care \& Population Health, UCL, London, UK; ${ }^{2}$ Cegedim Strategic Data Medical Research UK, Cegedim Strategic Data, London, UK

Background Since the thalidomide tragedy in the 1960s, many women are concerned with the use of drugs in pregnancy. Antiepileptic drugs (AEDs) are commonly used to treat epilepsy and bipolar disorders in women of child bearing age. However, some AEDs are associated with an increased risk of major congenital malformations. Untreated epilepsy and bipolar disorders in pregnancy may lead to devastating consequences for both mother and child. This leaves women and health care professionals with a dilemma as whether to continue AED medication in pregnancy. Using data from THIN, a UK primary care database, we examined pregnancy as a determinant for ceasing AED treatment.

Methods A cohort study of pregnant women receiving AEDs in UK primary care was conducted. We identified women who were prescribed AEDs in the three months before pregnancy. Time to last consecutive AED prescription in pregnancy was estimated whereby discontinuation of therapy was defined by a gap of more than three months between prescriptions. We identified a control group of twice as many non-pregnant women receiving AEDs. These women were randomly selected, but stratified within five year age bands and matched on indication for AEDs as for the pregnant women Cox's regression was used to compare the likelihood of discontinuing AEDs between pregnant and non-pregnant women.

Results In total, 934 women were prescribed AEDs three months before pregnancy. Pregnant women with epilepsy were twice as likely to cease AEDs compared to non-pregnant women (Hazard Ratio (HR):2.00, 95\% confidence interval (CI:1.62-2.47). Of 745 women with epilepsy, 601 (80.7\%) continued treatment into pregnancy and $465(62.4 \%)$ to the end of the second trimester. Of 1,490 non-pregnant women with epilepsy, 1,242 (83.4\%) and 1,071 (71.9\%) continued for comparable time periods.

Pregnant women with bipolar disorder or depression were three times as likely to cease AEDs compared to non-pregnant women (HR:3.07, 95\% CI:2.04-4.62). Of 54 pregnant women with bipolar disorder, $27(50.0 \%)$ continued into pregnancy, and only $8(14.8 \%)$ to the end of the second trimester. In 108 non-pregnant women with bipolar disorder, 82 (75.9\%) and 58 (53.7\%) continued for comparable periods.

Conclusion Pregnancy is a determinant for the discontinuation of AEDs during pregnancy, especially in women with bipolar disorder or depression despite the potentially severe consequences associated with not treating the underlying illness in pregnancy.

\section{PS26 PREVALENCE AND ASSOCIATIONS OF LIMITED HEALTH LITERACY IN CHRONIC KIDNEY DISEASE: A SYSTEMATIC REVIEW}

doi:10.1136/jech-2012-201753.125

${ }^{1}$ SDS Fraser, 'PJ Roderick, 'M Casey, ${ }^{2} \mathrm{MW}$ Taal, 'HM Yuen, ${ }^{3} \mathrm{D}$ Nutbeam. ${ }^{1}$ Public Health Sciences and Medical Statistics, University of Southampton, Southampton, UK; ${ }^{2}$ Renal Medicine, Royal Derby Hospital, Derby, UK; ${ }^{3}$ Vice Chancellor, University of Southampton, Southampton, UK

Background Chronic kidney disease (CKD) is a prevalent and growing problem, strongly associated with obesity, diabetes, hypertension, and cardiovascular disease. Health inequalities are recognised throughout the CKD pathway, including prevalence of risk factors, prevalence of $\mathrm{CKD}$, progression, and renal replacement therapy. There is evidence that an adequate level of health literacy (defined as the cognitive and social skills which determine the motivation and ability of individuals to gain access to, understand, and use information in ways that promote and maintain good health') contributes to improved disease management, and that inadequate health literacy is a potentially modifiable determinant of poor health outcomes and health inequalities in people with chronic disease. The aim of this review was to synthesise and critically appraise the literature evidence on the prevalence and associations of limited health literacy in CKD.

Methods Seven databases were searched using terms for CKD and health literacy $(\mathrm{HL})$. Cross sectional studies, and baseline data from cohort and randomised controlled trials were included where they assessed and presented the prevalence of limited HL using a validated tool in adults with CKD of any stage. The primary outcome was an objectively measured prevalence of limited HL in a population with CKD. The secondary outcome was associations of limited HL. Study quality was assessed by two reviewers using standardised criteria. Prevalence values were combined using a random effect model to give overall prevalence.

Results 82 studies were identified from searching, of which six met the inclusion criteria. The total number of people with CKD in all studies was 1,405. Five studies were in dialysis or transplant populations, and all were from the US. There was significant heterogeneity in the prevalence of limited HL $(9 \%$ to $32 \%$ (median $25 \%$, inter-quartile range $16 \%)$ ). The pooled prevalence of limited 